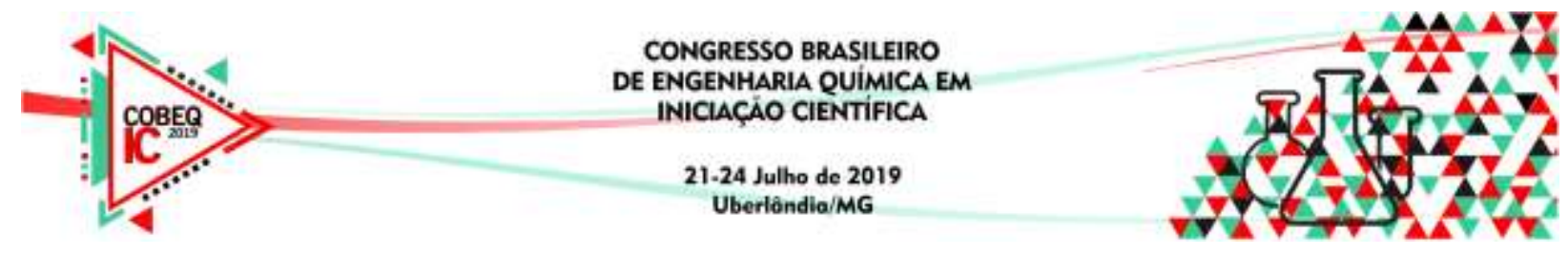

\title{
DEGRADAÇÃo DA CITARABINA PELO MÉTODO SONO ELETROQUÍMICO FOTO-ASSISTIDO EM MEIO DE URINA ARTIFICIAL
}

\author{
A.C.M.BORGES ${ }^{1}$, F.S.PIMENTA ${ }^{1}$, SOUZA,E.S.C. ${ }^{1}$, R.ANTONELLI ${ }^{2}$, G.R.P.MALPASS ${ }^{1}$ \\ ${ }^{1}$ Universidade Federal do Triângulo Mineiro, Departamento de Engenharia Química \\ ${ }^{2}$ Universidade Estadual de Campinas, Departamento de Engenharia Química \\ E-mail para contato: fernandapimentasssp@hotmail.com
}

\begin{abstract}
RESUMO - O aumento no número de pessoas diagnosticadas com câncer é realidade vivenciada em várias unidades de saúde. Nesse contexto, os resíduos provenientes dos tratamentos oncológicos necessitam de maior atenção, uma vez que possuem um elevado potencial de toxicidade e esse efeito negativo não é atenuado em estações de tratamento convencionais. Além disso, o quimioterápico mais utilizado em leucemias é a Citarabina, classificado como um micropoluente, pois é eliminado por pacientes que estão em tratamento através de excretas como a urina e fluidos corporais. A contaminação humana com compostos desse tipo pode ocorrer através da inalação, pela pele e pelas mucosas. Diante disso, o presente estudo visa avaliar a viabilidade da combinação das técnicas: eletroquímica, fotoquímica e sonoquímica na degradação desses fármacos presentes na urina de pacientes. As análises foram realizadas em uma célula eletroquímica de bancada com fluxo contínuo e avaliou-se a porcentagem de remoção de carbono orgânico total das amostras. As condições ótimas de degradação encontradas são 1,42A para a corrente, $1,73 \mathrm{~mL} / \mathrm{min}$ para a vazão da bomba e 198 minutos. Em suma, a avaliação por UPLC-MS corroborou com os dados obtidos no planejamento e constatou-se a remoção de Ancitabina, um percursor da Citarabina, contudo pode-se afirmar que foi obtido êxito no processo de degradação do quimioterápico através do método escolhido.

Palavras-chave: Efluente hospitalar, quimioterápicos, toxidade, Citarabina.
\end{abstract}

\section{INTRODUÇÃO}

Os resíduos de serviços de saúde que englobam os resíduos provenientes de hospitais, clinicas e laboratórios patológicos aumentaram consideravelmente nos últimos anos (Moura e Silva, 2016). Estes vem sendo constantemente inseridos no ambiente aquático por meio da disposição inadequada oriunda de indústrias farmacêuticas, efluentes hospitalares não tratados e através da excreção de fármacos pelos inúmeros consumidores. Além disso, os medicamentos citostáticos e metabólitos excretados pelos pacientes são descarregados diretamente no sistema de esgoto sem controle especifico depois de serem administrados nos hospitais. Embora o esgoto sofra grande diluição, é importante ressaltar que mesmo em baixas concentrações a presença desses fármacos em mananciais é um risco para o habitat aquático e para a saúde humana, reforçando a necessidade de tratamento especifico por parte das clinicas 


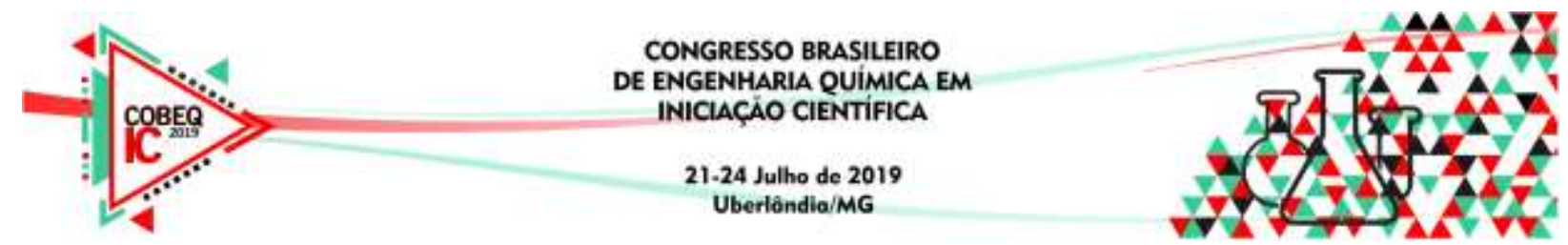

de oncologia. Diante do exposto, o presente estudo propõe a utilização do processo sonoeletroquímico foto-assistido para promover a remoção/degradação do quimioterápico citarabina, o fármaco mais utilizado no tratamento de leucemias, em meio a urina artificial investigando a eficiência desse processo no tratamento de efluentes e águas residuais.

\section{REVISÃO BIBLIOGRÁFICA}

Apesar da periculosidade associada aos resíduos utilizados em tratamentos de quimioterapia, pesquisas realizadas demonstram que em países como o Brasil, de todos os hospitais, apenas 228 mil toneladas de resíduos de serviços de saúde foram coletadas em 2010, sendo que a coleta destes resíduos executada por grande parte dos municípios é parcial. Desse modo, ocorre o desconhecimento sobre a quantidade total destes resíduos, levantandose questionamentos acerca de uma possível destinação incorreta, gerando um impacto ambiental considerável ao ecossistema (Oliveira, 2011). Além disso, uma das vias de introdução destes fármacos no meio ambiente é por meio do descarte inadequado de quimioterápicos com validade expirada e através dos resíduos contidos nas embalagens utilizadas em unidades hospitalares (Moura e Silva, 2016). Entretanto, a principal via de inserção de fármacos nos ecossistemas é por meio das excretas de pacientes. Alguns estudos tem demonstrado que a eletrólise poderia eliminar completamente a citotoxidade, mutagenicidade e atividade antibacteriana das águas residuais clinicas contendo antineoplásicos (Hirose et al, 2005). Na literatura encontra-se alguns estudos que aplicaram diversos métodos a fim de decompor o fármaco citarabina, representada pela Figura 1, no meio aquático, por ozonólise, oxidação fotoquímica, processo eletroquímico, $\mathrm{H} 2 \mathrm{O} 2$ / UV, degradação fotocatalítica na presença de $\mathrm{TiO}_{2}$ e carbono ativo.

Figura 1- Estrutura química da citarabina.

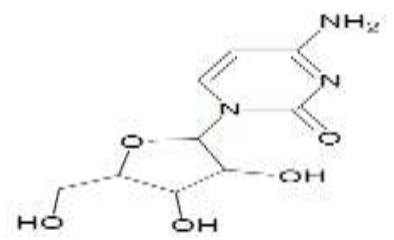

Fonte: Produzida no ChemSketch, 2017

Entre as técnicas de degradação apresentadas na literatura se destacam a eletroquímica foto-assistida e sonoeletroquímica. Um estudo mostrou a eficácia da técnica eletroquímica foto-assistida com energia ultrassônica para a remoção de pesticidas (Pinto et al.,2017; Antonelli et al.;2017). Com base em informações coletadas nos hospitais da cidade de Uberaba - MG (Hospital de Clínicas da UFTM e Hospital Dr. Hélio Angotti), dentre a gama de quimioterápicos existentes, a citarabina está entre os mais utilizados. Desta forma, esse trabalho visa à degradação desse quimioterápico.

\section{OBJETIVO}




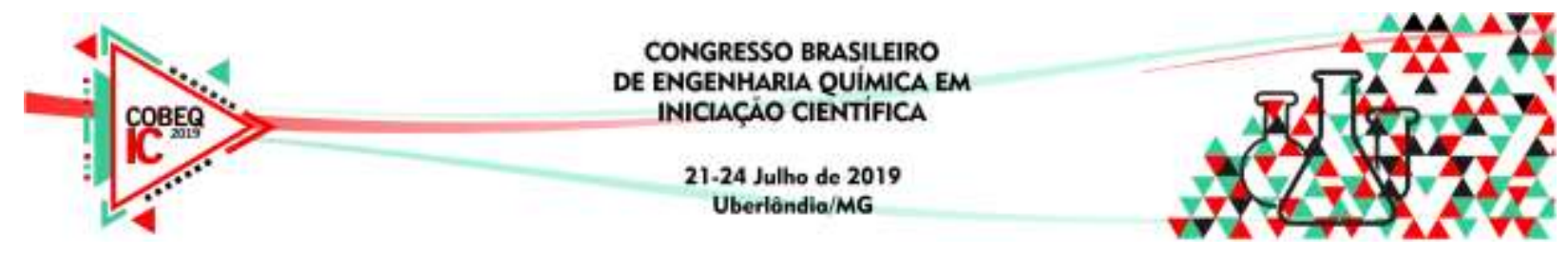

Esse trabalho tem como objetivo estudar a viabilidade da degradação do fármaco citarabina em meio à urina artificial pelo processo sonoeletroquímico foto-assistido, assim como determinar as variáveis significativas e as condições ótimas para a degradação.

\section{METODOLOGIA}

O delineamento experimental e a análise dos dados foram efetuados utilizando o software STATISTICA. A Citarabina foi analisada por UPLC-MS (Cromatografia líquida com espectrômetro de massa) feita em parceria com a empresa Ourofino Agrociência em Uberaba-MG e as análises de carbono orgânico total (COT) foram realizadas em parceria com o Laboratório de Desenvolvimento de Tecnologias Ambientais (LDTAmb), do IQSC/USP. Os experimentos foram realizados por meio do planejamento fatorial $2^{3}$, com três variáveis: vazões da bomba (3,24 e 4,67 mL/min), corrente aplicada ( 0,5 e 1 A) e tempo de reação (30 e $60 \mathrm{~min}$ ) e também realizou-se o planejamento composto central (PCC) para investigar a influência das variáveis sobre a mineralização das amostras, por meio da porcentagem de remoção de carbono orgânico total (COT). Nesse estudo utilizou-se uma célula eletroquímica de bancada com fluxo contínuo através de uma bomba peristáltica. A célula foi montada com dois espaçadores de Viton e Teflon com espassamento de 4,36 milimetros, representada na Figura 2.

Figura 2- Célula eletroquímica em fluxo contínuo: (a) Visão explodida: (1) Chapa externa de aço; (2) Espaçadores de Teflon; (3) Espaçadores de Viton; (4) Janela de quartzo; (5) Cátodo (Ti); (6) Ânodo e (7) Suporte. (b) Célula montada. (c) Sistema utilizado, sendo: (1) banho ultrassom; (2) reservatório da solução efluente; (3) fonte luminosa de radiação UV; (4) bomba; (5) célula eletroquímica; (6) fonte estabilizada
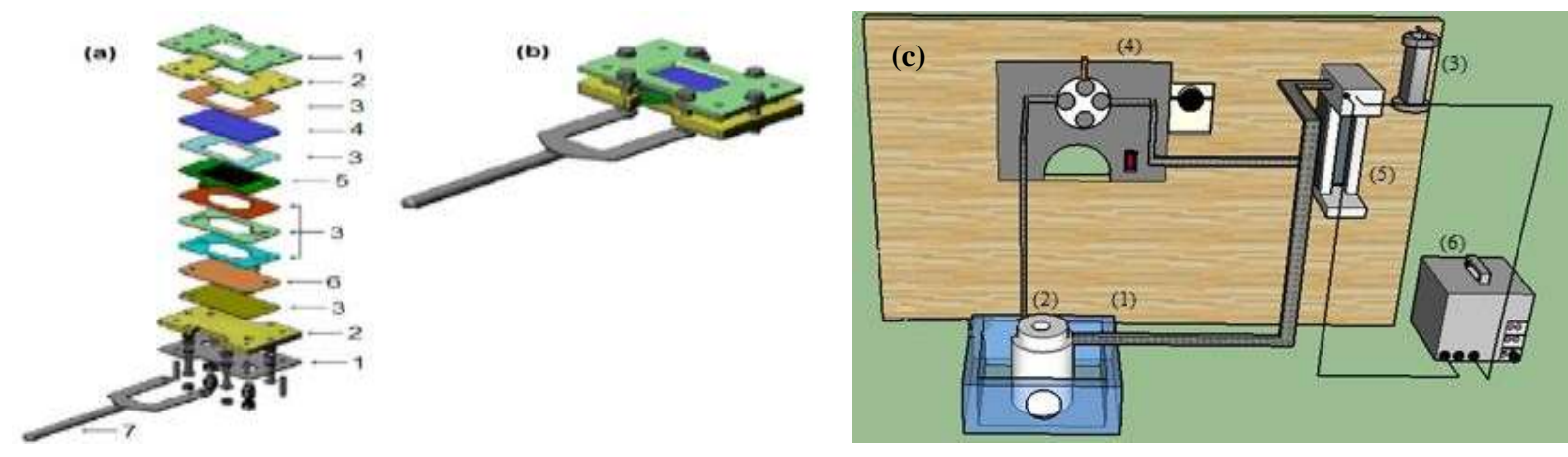

Fonte: Elaborado pela autora, 2017.

Além disso, empregou-se uma fonte luminosa ultravioleta de $375 \mathrm{~W}$ inserida diretamente na célula e foi utilizado uma cuba ultrassônica com frequência de $42 \mathrm{kHz}$. Em complemento, a urina preparada artificialmente foi utilizada nesse estudo, pois ela representa um interessante meio quando se pretende simular um determinado comportamento em urina natural, uma vez que possui a composição muito próxima da verdadeira (Parra, 2013). A concentração de citarabina utilizada foi de $3 \mathrm{mg} / \mathrm{L}$, sendo o volume total do efluente tratado de $300 \mathrm{~mL}$. A concentração do fármaco foi definida fazendo considerações sobre a área de 


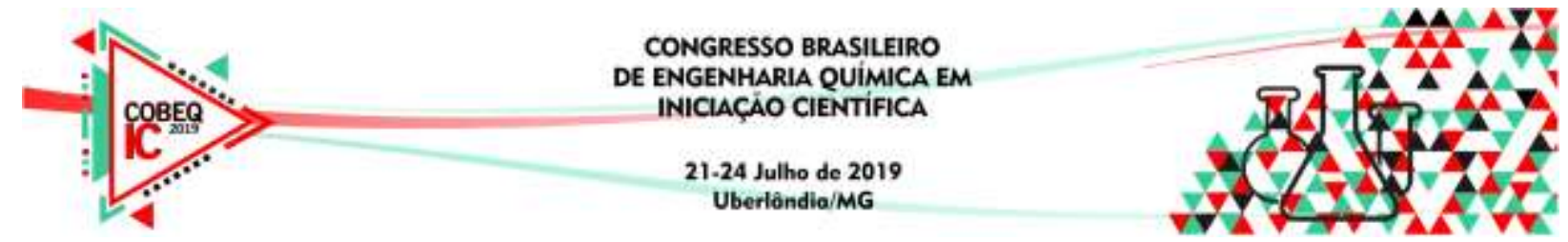

superfície corporal média de um paciente $\left(1,72 \mathrm{~m}^{2}\right)$ e considerando que são inseridos 100 mg. $\mathrm{m}^{2}$ do fármaco no paciente, sendo que $10 \%$ do fármaco é excretado de forma inalterada e que é consumido um valor médio de 400 litros de água por leito em um dia. Além disso, considerou-se que um hospital cuida de cerca de 70 pacientes por dia.

\section{RESULTADOS}

Utilizou-se a remoção de COT (Carbono orgânico total) como variável resposta do planejamento fatorial, para analisar a mineralização de todos os poluentes orgânicos presentes no efluente estudado. Além das análises de COT, efetuou-se análises de pH, obtendo-se valores na faixa de 6,50 a 6,80 depois dos ensaios de degradação, valores estes dentro dos limites estabelecidos pela Resolução CONAMA $n^{\circ}$ 357. Além disso, as variáveis independentes e dependentes foram analisadas pelo software STATISTICA com um intervalo de confiança de $95 \%$. Com o modelo estatístico determinado, construiu-se o gráfico de pareto para análise das variáveis com os efeitos mais significativos, Figura 3.

Figura 3- Gráfico de pareto para o planejamento fatorial.

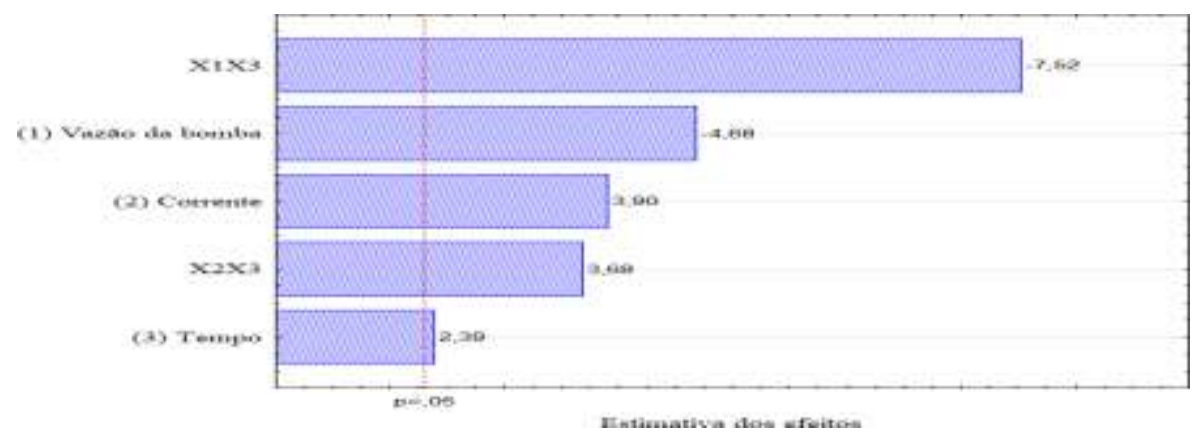

Fonte: Elaborado pela autora, 2017

A partir do gráfico de pareto é possível constatar que a vazão da bomba tem efeito negativo na resposta. Já a corrente elétrica apresenta influência positiva e a variável tempo é a que apresenta menor significância dentre as variáveis estudadas e possui influência positiva na resposta. Em adição ao mencionado, o comprimento de onda característico da citarabina é 272 nm no UV-VIS, assim, após os ensaios de degradação observou-se pelas análises no espectrofotômetro que houve sobreposição de picos, podendo ser característicos da produção de cloro livre (com comprimento de onda em $290 \mathrm{~nm}$ ). Além disso, através da análise por UPLC-MS (cromatografia líquida com espectrômetro de massa) constatou-se que provavelmente existe ancitabina no meio, o que implica que será necessário um tempo de degradação muito maior para poder obter-se a conversão da ancitabina em citarabina e a posterior degradação dessa. Além disso, a melhor condição de degradação é a que possui o melhor COT removido (21,95\%). Assim sendo, a avaliação por UPLC-MS corrobora com os dados obtidos pelo planejamento experimental que mostra que, quanto menor a vazão e maior a corrente elétrica melhor é a remoção de COT. Desse modo, estudos mostram que a presença da ancitabina, em amostras de citarabina, é muito útil para manter-se doses efetivas de 


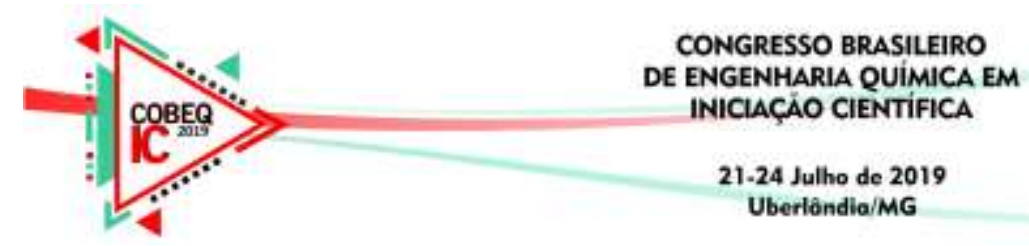

citarabina, uma vez que essa sofre deaminação rápida no organismo para o uracil arabinosídeo, segundo Kirsch e Notari (1984). A Tabela 1, representa os ensaios de remoção de carbono orgânico total (COT) através do planejamento composto central.

Tabela 1- Resultados de remoção de COT do planejamento composto central.

\begin{tabular}{|c|c|c|c|c|}
\hline $\begin{array}{c}\text { Amostra } \\
\mathbf{s}\end{array}$ & $\begin{array}{c}\text { Corrente } \\
\text { elétrica (A) }\end{array}$ & $\begin{array}{c}\text { Tempo } \\
(\mathbf{m i n})\end{array}$ & $\begin{array}{c}\text { COT } \\
(\mathbf{m g} / \mathbf{L} \\
)\end{array}$ & $\begin{array}{c}\text { Remoção } \\
\text { COT (\%) }\end{array}$ \\
\hline $\mathbf{1}$ & $1,5(-1)$ & $30(-1)$ & 4420 & 18,14 \\
\hline $\mathbf{2}$ & $1,5(-1)$ & $90(1)$ & 4160 & 22,96 \\
\hline $\mathbf{3}$ & $2,5(+1)$ & $30(-1)$ & 4440 & 17,78 \\
\hline $\mathbf{4}$ & $2,5(+1)$ & $90(+1)$ & 3880 & 28,15 \\
\hline $\mathbf{5}$ & $1,4(-\alpha)$ & $60(0)$ & 3930 & 27,22 \\
\hline $\mathbf{6}$ & $2,6(+\alpha)$ & $60(0)$ & 4160 & 22,96 \\
\hline $\mathbf{7}$ & $2,0(0)$ & $23,7(-\alpha)$ & 4520 & 16,30 \\
\hline $\mathbf{8}$ & $2,0(0)$ & $96,3(+\alpha)$ & 4020 & 25,55 \\
\hline $\mathbf{9}(\mathbf{C})$ & $2,0(0)$ & $60(0)$ & 4310 & 20,18 \\
\hline $\mathbf{1 0}(\mathbf{C})$ & $2,0(0)$ & $60(0)$ & 4330 & 19,81 \\
\hline $\mathbf{1 1}(\mathbf{C})$ & $2,0(0)$ & $60(0)$ & 4320 & 20,00 \\
\hline $\mathbf{1 2}(\mathbf{C})$ & $2,0(0)$ & $60(0)$ & 4350 & 19,44 \\
\hline
\end{tabular}

Fonte: Elaborado pela autora, 2018

O ponto ótimo para a degradação foi obtido pelo software STATISTICA e equivale à 1,42 A para a corrente, $1,73 \mathrm{~mL} / \mathrm{min}$ para a vazão da bomba e 198 minutos para o tempo. Ademais, a presença da creatinina e ureia presentes na urina, interferem no processo eletroquímico, devido à diminuição dos sítios eletroativos do eletrodo. Esse fenômeno reduz a eficiência dos processos e dificulta a degradação de moléculas específicas em resíduos naturais. Além disso, através de experimentos envolvendo a densidade de corrente, Equação 1 , pode-se concluir que é preferível trabalhar com valores maiores de tempo e com densidades de correntes menores, sendo que no ponto com baixa vazão da bomba apresentou-se um valor de EC (eficiência de corrente) mais elevado.

$$
E C(\%)=\frac{Q_{C I}}{Q_{T}} \cdot 100
$$

Além disso, através de alguns experimentos concluiu-se que o consumo energético está diretamente relacionado à carga aplicada, assim quanto maior o tempo e/ou a corrente aplicada para se atingir determinada remoção, maior será o consumo energético (CE), Equação 2.

$$
C E=\frac{i \cdot U \cdot t}{1000 . V}
$$




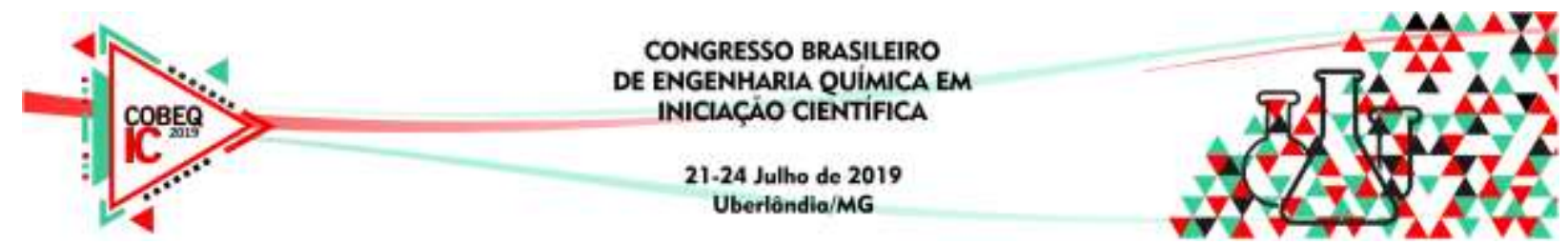

\section{CONCLUSÃO}

Com os ensaios realizados definiu-se que as condições ótimas de remoção de carbono orgânico total (COT) pelo método sonoeletroquímico foto-assistido são: 1,4 A para a corrente, $1,73 \mathrm{~mL} / \mathrm{min}$ para a vazão da bomba e 198 minutos para o tempo. A variável mais significativa no processo de degradação foi a vazão da bomba que apresenta influência negativa, seguida da corrente elétrica, com influência positiva na resposta. A terceira variável significativa foi o tempo de reação, com influência positiva. Além disso, pela análise por UPLC-MS infere-se que o processo resultou na remoção da ancitabina, percursor da citarabina, o que sugere que será necessário um tempo maior para obter degradação da citarabina. Desse modo, a avaliação por UPLC-MS corrobora com os dados obtidos pelo planejamento experimental, no entanto vale ressaltar que a ancitabina se converte no organismo em citarabina. Em complemento, os resultados indicaram que a utilização de urina como eletrólito suporte eleva o teor de COT no meio e interfere no processo eletroquímico, devido a diminuição dos sítios eletroativos do eletrodo. Desse modo, é observada significativa remoção dos compostos orgânicos presentes no efluente mesmo em um meio mais complexo como o de urina artificial.

\section{REFERÊNCIAS}

HIROSE J, KONDO F, NAKANO T, KOBAYASHI T, HIRO N, ANDO Y, et al.Inactivation of antineoplastics in clinical wastewater by electrolysis. Chemosphere, v.60, p.1018-24, 2005.

KIRSCH, L.; NOTARI, R. E. Aqueous conversion kinetics and mechanisms of ancitabine, a prodrug of the antileukemic agent cytarabine. Journal of Pharmaceutical Science, 1984, 73, 896-902.

MOURA, L. L. e SILVA, R. F. Medicamentos antineoplásicos no meio ambiente: a contribuição de um hospital universitário de alta complexidade. Revista gestão \& sustentabilidade ambiental, Florianópolis, v. 5, n. 1, p. 313-333, 2016.

OLIVEIRA, L. C. (2011). Uso integrado do método QFD e de técnicas estatísticas de planejamento e análise de experimentos na etapa do projeto do produto e do processo. Belo Horizonte. 270p. Dissertação (Mestrado) - Universidade Federal de Minas Gerais.

PARRA, K. N. Degradação eletroquímica de tetraciclina em meio de urina artificial. 2013. 90 f. Dissertação de mestrado. Instituto de Química de São Carlos, Universidade de São Paulo. 2013.

PINTO, C. F.; ANTONELli, R. A.; ARAÚJO, K. S.; FORNAZARI, A. L. T.; FERNANDES, D. M.; GRANATO, A. C.; AZEVEDO, E. B.; MALPASS, G. R.P. (2017) Experimental-design-guided approach for the removal of atrazine by sono-electrochemicalUVchlorine techniques. Environmental Technology, doi: 10.1080/09593330.2017.1395480 